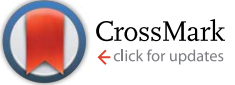

Cite this: RSC Adv., 2017, 7, 15736

Received 25th December 2016

Accepted 6th March 2017

DOI: 10.1039/c6ra28673a

rsc.li/rsc-advances

\section{Co-assembly of Fmoc-tripeptide and gold nanoparticles as a facile approach to immobilize nanocatalysts $\uparrow$}

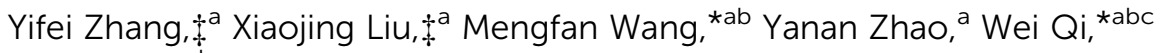 \\ Rongxin $\mathrm{Su}^{\mathrm{abc}}$ and Zhimin $\mathrm{He}^{\mathrm{a}}$
}

\begin{abstract}
A facile approach was developed to immobilize gold nanoparticles (AuNPs) onto peptide nanofibers for catalytic applications. In this study, fluorenylmethoxycarbonyl diphenylalanine (Fmoc-FF) was conjuncted with histidine, arginine or cysteine at their C-terminus to provide binding sites for AuNPs. The coassembly of Fmoc-tripeptide and AuNPs was achieved by dropping the peptide monomer solution directly into the AuNP solution, leading to the formation of nanofibers and the immobilization of AuNPs in one step. Atomic force microscopy, transmission electron microscope and circular dichroism analyses demonstrated that the presence of AuNPs does not significantly change the morphology and secondary structure of the nanofibers. The histidine-containing peptide-immobilized AuNPs were found to display favorable catalytic activity and stability for the reduction of 4-nitrophenol to 4-aminophenol. The present approach to fabricating nanomaterial-supported AuNPs may be extended to the production of other nanoparticle-containing composites in the fields of catalysis, sensing and biomedicine.
\end{abstract}

\section{Introduction}

Over the past few decades, nanocatalysis has become a rapidly growing field that involves the use of nanomaterials as catalysts for a variety of homogeneous and heterogeneous reactions..$^{\mathbf{1 4}}$ Among these materials, gold nanoparticles (AuNPs) have attracted considerable attention because of their excellent applications in carbon monoxide oxidation, hydrogen production and pollutant degradation..$^{5-10}$ On one hand, the large surface-to-volume ratio endows AuNPs with high activity. On the other hand, the small size of AuNPs inevitably leads to agglomeration, which seriously suppresses their catalytic activity. To conquer this problem, many efforts have been undertaken to immobilize AuNPs onto solid organic or inorganic supports. Dotzauer et al. immobilized AuNPs in a multilayer polyelectrolyte film via layer-by-layer adsorption supported by porous supports, including alumina and polycarbonate. ${ }^{11}$ Fang et al. reported an approach to simultaneously synthesize and immobilize AuNPs in

${ }^{a}$ State Key Laboratory of Chemical Engineering, School of Chemical Engineering and Technology, Tianjin University, Tianjin 300072, P. R. China. E-mail: mwang@tju. edu.cn; qiwei@tju.edu.cn

${ }^{b}$ Tianjin Key Laboratory of Membrane Science and Desalination Technology, Tianjin 300072, P. R. China

'The Co-Innovation Centre of Chemistry and Chemical Engineering of Tianjin, Tianjin 300072, P. R. China

$\dagger$ Electronic supplementary information (ESI) available: UV spectra in the range of 250-310 nm, Congo red reduction reaction, images before and after $72 \mathrm{~h}$ incubation. See DOI: 10.1039/c6ra28673a

$\ddagger$ These authors contributed equally to this work. electrospun polyethyleneimine (PEI)/polyvinyl alcohol (PVA) nanofibers. ${ }^{12}$ Moreover, natural materials are also advisable for immobilization of nanoparticles. Niu et al. deposited AuNPs on cellulose nanofibers of filter paper to yield bulk cellulose-based catalytic membranes, which exhibited excellent catalytic activities toward the reduction of 4-nitrophenol. ${ }^{13}$ Lin et al. reported the biological synthesis and binding of AuNPs to the cell surface of yeast Pichia pastoris (P. pastoris), which exhibited high affinity for $\mathrm{Au}(\mathrm{III})$ species in aqueous solution. ${ }^{\mathbf{1 4}}$

Recently, molecular self-assembly has been developed as a popular tool for constructing micro- and nano-structural supports. Low-molecular-weight gels are known as distinct soft materials that are obtained through bottom-up fabrication of small molecules into various types of nano-fibril, strand or tape structures. Therefore, the development of self-assembled architectures from small molecules, including some biological molecules, provides a promising platform for nanoscaled applications. Peptides, especially some aromatic peptides and their derivatives, are well known building blocks for the fabrication of peptide-based nanomaterials because their assembly can be easily conducted under the driving force of $\pi-\pi$ stacking between aromatic groups. ${ }^{15}$ Because these materials combine the simplicity of small molecules with the versatility of peptides, they make excellent nano-carriers or supports in the fields of drug delivery, tissue engineering and chem-biosensing. ${ }^{16-18}$

In this study, a peptide-based nanomaterial was developed as a novel support to immobilize AuNPs. Diphenylalanine (FF) is the simplest peptide that can self-assemble into nanotubes in pure water through $\pi-\pi$ interactions between the aromatic side 


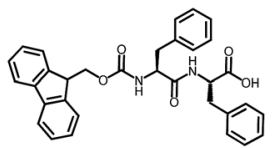

Fmoc-FF

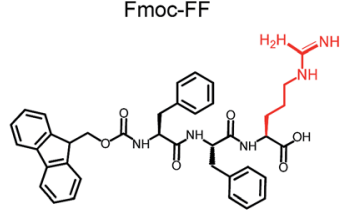

Fmoc-FFR

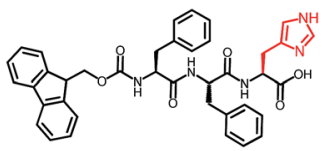

Fmoc-FFH

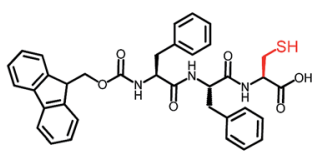

Fmoc-FFC
Scheme 1 Molecular structures of Fmoc-FF, Fmoc-FFH, Fmoc-FFR and Fmoc-FFC.

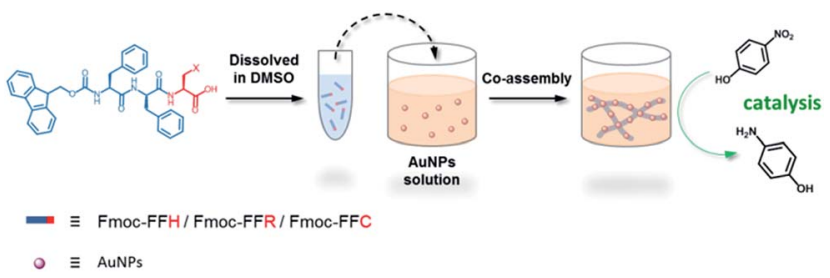

Scheme 2 One-step immobilization of AuNPs through co-assembly of peptide and AuNPs.

chains. ${ }^{19}$ Fluorenylmethoxycarbonyl (Fmoc) is a commonly used protecting group in peptide synthesis whose aromatic fluorenyl ring can further enhance $\pi-\pi$ stacking and promote the formation of supramolecular architectures. ${ }^{15,20}$ Therefore, Fmoc-FF was chosen as the basic scaffold to build the nanosupport. Additionally, it has been reported that many amino acid residues can be attached to metal nanoparticles through different types of interactions. The histidine tag $\left(\mathrm{His}_{6}\right)$ in biotechnology can directly couple to AuNPs via $\mathrm{N} \varepsilon-\mathrm{Au}$ interactions. ${ }^{21}$ The positively charged amino acids (arginine and lysine) or the negatively charged amino acids (aspartic acid and glutamic acid) can easily combine with opposite-charged nanoparticles through electrostatic attraction. Moreover, the sulfhydryl group on cysteine can form a strong covalent interaction with AuNPs through the formation of a S-Au bond. ${ }^{22}$ Herein, Fmoc-FF was further modified to conjugate histidine, arginine and cysteine at their C-terminus to provide a practical binding site for AuNPs (Scheme 1). The co-assembly of AuNPs and peptide (Fmoc-FFH, Fmoc-FFR or Fmoc-FFC) was achieved by dropping the peptide monomer solution directly into the AuNPs solution, resulting in the formation of the nano-support and the immobilization of AuNPs in one step (Scheme 2). The physical properties of the peptide-immobilized AuNPs were characterized and their catalytic activities were evaluated through the reduction of 4-nitrophenol and Congo red.

\section{Experimental section}

\section{Materials}

Fmoc-Phe-Phe-His-COOH (Fmoc-FFH), Fmoc-Phe-Phe-Arg$\mathrm{COOH}$ (Fmoc-FFR) and Fmoc-Phe-Phe-Cys-COOH (Fmoc-FFC) were purchased from GL Biochem Ltd. (Shanghai, China). $\mathrm{HAuCl}_{4}$, dimethyl sulfoxide (DMSO), 4-nitrophenol (4-NP) and other chemicals and solvents were commercially purchased.

\section{Synthesis of AuNPs}

AuNPs were prepared by citrate reduction of $\mathrm{HAuCl}_{4}$. An aqueous solution of $\mathrm{HAuCl}_{4}(1 \mathrm{~mL}, 1 \mathrm{wt} \%)$ was added to ultrapure water $(100 \mathrm{~mL})$. After gently stirring for $1 \mathrm{~min}$, an aqueous solution of sodium citrate ( $1 \mathrm{~mL}, 1 \mathrm{wt} \%$ ) was added. Then, $1 \mathrm{~mL}$ of $\mathrm{NaBH}_{4}$ solution containing $0.075 \mathrm{wt} \%$ sodium citrate and 1 $\mathrm{wt} \%$ sodium citrate was quickly added to the mixture. Stirring was continued for $5 \mathrm{~min}$ and the resulting AuNPs solution was stored at $4{ }^{\circ} \mathrm{C}$.

\section{Co-assembly of peptides and AuNPs}

A stock solution was prepared by dissolving $5 \mu \mathrm{mol}$ of peptides (Fmoc-FFH, Fmoc-FFR or Fmoc-FFC) in $50 \mu \mathrm{L}$ of DMSO. Then, a certain amount of stock solution was dropped into $1 \mathrm{~mL}$ of asprepared AuNPs solution so that the final concentration of peptide was $5 \mathrm{mM}$. The mixture was gently shaken and then kept still at room temperature until CoA-H-AuNPs, CoA-RAuNPs and CoA-C-AuNPs were obtained. For the preparation of self-assembled peptides, the same peptide stock solution was dropped into ultrapure water instead of AuNPs solution. The mixture was gently shaken and then kept still at room temperature to obtain SA-H, SA-R and SA-C. All the samples were washed with ultrapure water so as to remove the free AuNPs.

\section{Characterization}

The morphologies of the obtained CoA-X-AuNPs and SA-X were investigated using an Agilent 5500 atomic force microscopy (Agilent, USA). Transmission electron microscope (TEM) were recorded on JEOL $2100 \mathrm{~F}$ with $200 \mathrm{kV}$ accelerating voltages (JEOL Ltd., Japan). Circular dichroism spectra were obtained on a J810 CD spectropolarimeter (JASCO, Japan) with a $0.2 \mathrm{~mm}$ path length quartz cell at room temperature. The wavelength range was set to 260-190 $\mathrm{nm}$ and the scan speed was $100 \mathrm{~nm} \mathrm{~min}{ }^{-1}$. UV-Vis spectra were obtained on an SpectraMAX 190 ultravioletvisible spectrophotometer (Molecular device, USA) with a $1 \mathrm{~mm}$ path length quartz cell at room temperature. The wavelength range was 220-340 $\mathrm{nm}$. Thermogravimetric analysis (TGA) was performed on a simultaneous TGA-DTA apparatus (PTC-10A, Rigaku, Japan) with the same mass of the dry CoA-X-AuNPs samples. The temperature was programed from $25^{\circ} \mathrm{C}$ to $700{ }^{\circ} \mathrm{C}$ at the rate of $10^{\circ} \mathrm{C} \mathrm{min}^{-1}$.

\section{4-Nitrophenol reduction reaction}

4-NP (41.8 mg) and $\mathrm{NaBH}_{4}(56.7 \mathrm{mg})$ were dissolved in ultrapure water (100 and $5 \mathrm{~mL}$, respectively). As a representative example, $750 \mu \mathrm{L}$ of 4 -NP solution, $7 \mathrm{~mL}$ of ultrapure water and $100 \mu \mathrm{L}$ of peptide-immobilized AuNPs solution were pre-heated at $37{ }^{\circ} \mathrm{C}$ for $3 \mathrm{~min}$. Then, $1 \mathrm{~mL}$ of fresh $\mathrm{NaBH} 4$ solution was added to the mixture and stirred continuously. The reaction progress was monitored through determining the concentration of 4-NP on 
a SpectraMAX 190 spectrophotometer in the wavelength range of $200-500 \mathrm{~nm}$.

\section{Results and discussion}

\section{Characterization of peptide-immobilized AuNPs}

The self-assembly of peptides with the Fmoc-FF moiety is driven by hydrogen bonding and $\pi-\pi$ interactions between the aromatic fluorenyl rings and phenylalanine side chains. Short, aromatic peptides have been typically found to self-assemble into straight nanofibers or nanotubes through $\pi-\pi$ stacking, which can form unique 3D scaffolds and appear to be homogeneous hydrogels at the macroscopic scale. ${ }^{23}$ To determine whether the presence of AuNPs would affect the assembly of Fmoc-FFX ( $\mathrm{X}=\mathrm{H}, \mathrm{R}$ or $\mathrm{C})$ peptides, we investigated the gelation of Fmoc-FFH, Fmoc-FFR and Fmoc-FFC with or without AuNPs. As shown in Fig. 1 (top), all peptides can self-assemble into stable hydrogels in water in the absence of AuNPs. When the assembly was conducted in the presence of AuNPs, different phenomena were observed as shown in Fig. 1 (bottom). For the peptide conjuncted with histidine (Fmoc-FFH), the gelation time of $\mathrm{CoA}-\mathrm{H}-\mathrm{AuNPs}$ was prolonged to $60 \mathrm{~min}$, indicating that the interaction between AuNPs and Fmoc-FFH affected the assembly rate of the peptide monomer (Fig. 1, column 1, bottom). In the case of Fmoc-FFR, a reddish brown precipitate was generated immediately upon addition of Fmoc-FFR to the AuNPs solution, which remained insoluble for $24 \mathrm{~h}$ instead of forming a stable hydrogel. This result is attributed to the interaction between the positive arginine side chain and the negative AuNPs, which disrupts the electrostatic balance among the Fmoc-FFR molecules (Fig. 1, column 2, bottom). For the peptides conjuncted with neutral cysteine residue (Fmoc-FFC), the combination of AuNPs had little impact on the intrinsic force balance among peptides; hence, the addition of AuNPs did not affect the gelation of Fmoc-FFC (Fig. 1, column 3).

AFM and TEM were used to analyze the morphologies of the as-prepared SA-X and CoA-X-AuNPs, as shown in Fig. 2. Fibrous structures were obtained for all the SA-X and CoA-X-AuNP samples. Compared to SA-H nanofibers (Fig. 2A) with diameters

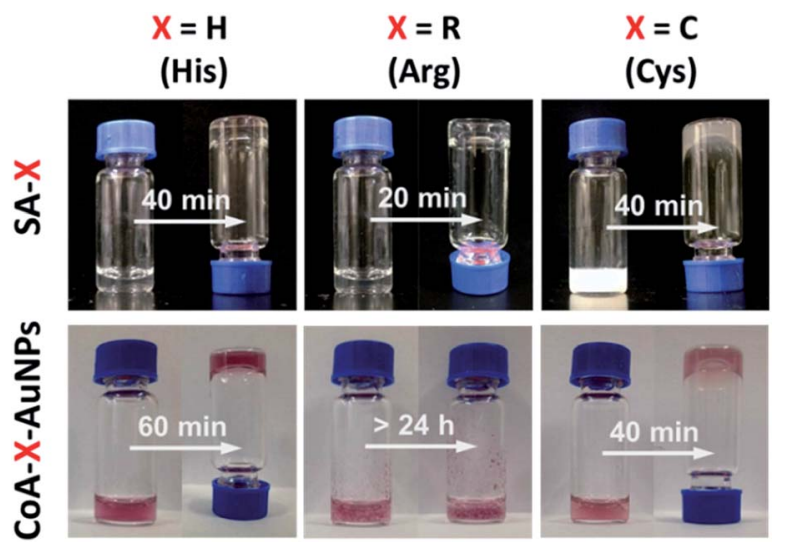

Fig. 1 Images of SA-H/COA-H-AuNPs (column 1), SA-R/COA-RAuNPs (column 2) and SA-C/CoA-C-AuNPs (column 3).
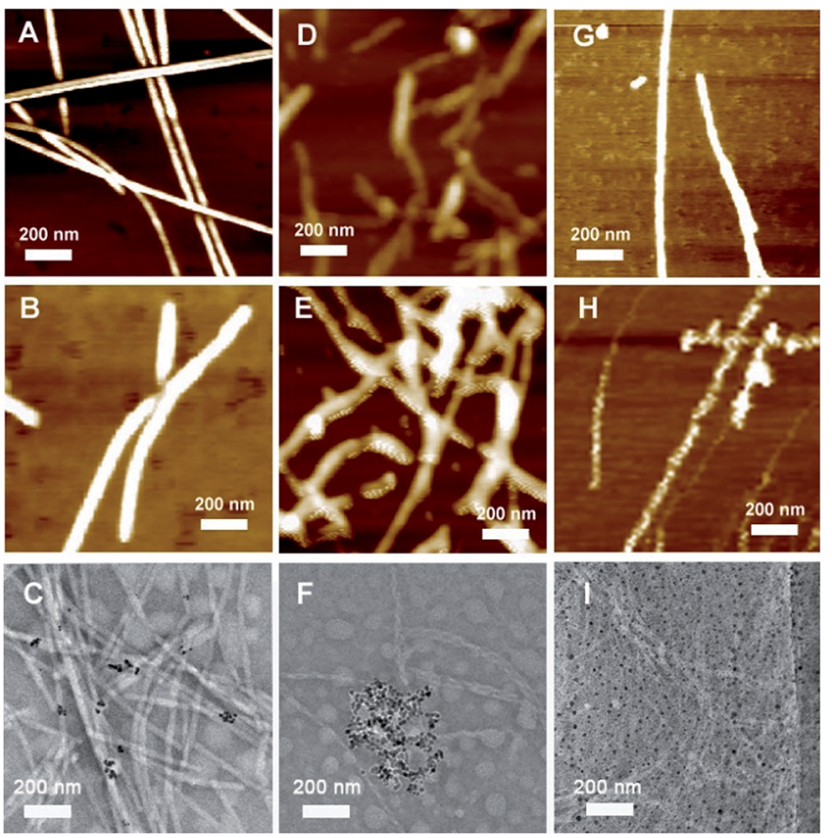

Fig. 2 AFM or TEM images of SA-H (A), COA-H-AuNPs (B, C), SA-R (D), CoA-R-AuNPs (E, F), SA-C (G) and CoA-C-AuNPs (H, I).

of approximately $20-30 \mathrm{~nm}$, the diameter of the CoA-H-AuNPs increased obviously (Fig. 2B), implying that the addition of AuNPs facilitated the formation of thicker nanofibers. However, the obtained CoA-H-AuNPs fibers were shorter than SA-H, which hinders the formation of 3D scaffolds and may explain the longer gelation time to become a hydrogel. The TEM image of CoA-H-AuNPs (Fig. 2C) demonstrated the association of the AuNPs with the peptide nanofibers. Since the concentration of AuNPs is low, not all fibers were observed to be decorated with them. For SA-R, the peptide monomers assembled into fragile fibrils because of the strong hydrophilicity of the arginine moiety (Fig. 2D). The introduction of AuNPs produced anomalous clusters attributed to the rapid neutralization of the negatively charged AuNPs and the positively charged arginine side chains (Fig. 2E). This result is in agreement with the formation of precipitates observed in Fig. 1 and the TEM result that the agglomeration of AuNPs was generated as shown in Fig. 2F. Unlike the others, the CoA-C-AuNPs display a rougher surface than SA-C (Fig. 2G and H), which implied that AuNPs can be attached to the peptide nanofibers. The TEM image (Fig. 2I) indicated that the AuNPs are distributed uniformly in CoA-C-AuNPs which might due to the strong interaction of SAu bond.

The immobilization of AuNPs on peptide nanofibers can also be determined using UV-Vis spectroscopy (Fig. 3A). For each peptide-immobilized AuNP sample, the spectrum was corrected using the corresponding SA-X nanofibers as a background, thus the observed absorbance at approximately $550 \mathrm{~nm}$ arises only from the localized surface plasmon resonance of the AuNPs. For CoA-H-AuNPs and CoA-C-AuNPs, a distinct and relatively sharp peak can be observed at $550 \mathrm{~nm}$; however, when the arginine-containing peptide was used to bind the AuNPs, 

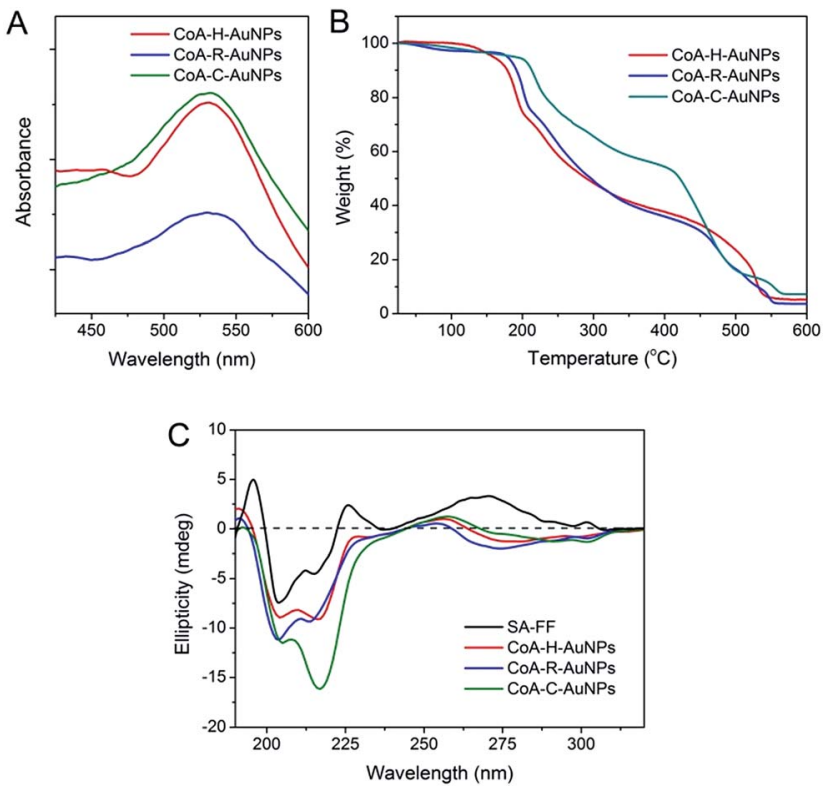

Fig. 3 UV-Vis spectra (A), TGA analysis (B) and circular dichroism spectra (C) of CoA-H-AuNPs, CoA-R-AuNPs and CoA-C-AuNPs.

a broader and red-shifted absorbance was observed. TGA was performed to investigate the relative amount of AuNPs conjuncted to different nanofibers as shown in Fig. 3B. Equal mass of CoA-X-AuNPs samples were gradually heated from room temperature to $700{ }^{\circ} \mathrm{C}$. Due to the loss of bound water, the weight of three samples decreased slowly before $240{ }^{\circ} \mathrm{C}$. The weight began to drop sharply beyond $240{ }^{\circ} \mathrm{C}$, which demonstrated the degradation of peptide nanofibers until $550{ }^{\circ} \mathrm{C}$. Based on the TGA curve, the AuNPs immobilized on $\mathrm{CoA}-\mathrm{H}-$ AuNPs, CoA-R-AuNPs and CoA-C-AuNPs could be approximately calculated as $4.2 \%, 3.4 \%$ and $7.2 \%$, respectively. The low amount of AuNPs in CoA-R-AuNPs might due to the formation of agglomerations which leads to the weak binding and prone to leaking from the peptide nanofibers. These results suggest that the type of amino acid X not only affect the polydispersity of AuNPs but also the amount of AuNPs immobilized on peptide nanofibers. In addition, the UV-Vis spectrum in the $250-310 \mathrm{~nm}$ region is typically assigned to the hydrophobic chromophore groups in the peptide molecules. The absorbance in this range was observed to decrease when AuNPs were attached to the peptide nanofibers (Fig. S1 in ESI $\dagger$ ). Compared to the corresponding samples without AuNPs, the maximum absorbance at $263 \mathrm{~nm}$ of CoA-H-AuNPs, CoA-R-AuNPs and CoA-C-AuNPs decreased $8.9 \%, 36.4 \%$ and $5.3 \%$, respectively, implying that the binding of AuNPs altered the original hydrophilic-hydrophobic microenvironment of the peptide nanofibers to different extents. Circular dichroism (CD) was utilized to give deep insight into the secondary structure of the peptide-immobilized AuNPs. As shown in Fig. 3C, the structure of all the CoA-XAuNPs exhibited a mixture of $\alpha$-helix and $\beta$-sheet (positive peak at approximately $185-200 \mathrm{~nm}$ and negative peaks at 204 and 216 $\mathrm{nm}$ ), which is in agreement with Ding's result that Fmoc-FF peptide self-assembled nanofibers (SA-FF) typically display a mixed structure. ${ }^{24}$ The derivation of Fmoc-FF and the presence of AuNPs did not appear to affect the basic secondary structure of the peptide nanofiber; thus, the Fmoc-FF moiety can be used as an appropriate platform to build nano-scaled supports.

\section{Catalytic activity of the peptide-immobilized AuNPs}

The catalytic activities of the as-prepared CoA-H-AuNPs, CoA$\mathrm{R}$-AuNPs and CoA-C-AuNPs were evaluated by using 4-NP as a substrate. 4-NP is a commonly used substrate because 4-NP can be catalytically converted to the colourless 4-nitroaniline which can be easily detected on UV-Vis spectrophotometer. Typical time-dependent UV-Vis absorption spectra were obtained during the reaction catalyzed by the peptideimmobilized AuNPs, which showed an obvious decrease in the absorbance peak of 4-NP at $400 \mathrm{~nm}$ (Fig. 4A and B) for CoA$\mathrm{H}$-AuNPs and CoA-R-AuNPs. As a result, the yellow reaction solutions became colorless after 25 and $35 \mathrm{~min}$ in the presence of CoA-H-AuNPs and CoA-R-AuNPs, respectively, indicating that the 4-nitrophenol had already been eliminated by the catalysts. However, we also found that the CoA-C-AuNPs lost all of their activity because the absorption of 4-NP remained constant during the reaction (Fig. 4C).

The activity difference of peptide-immobilized AuNPs originates from the different amino acid residues linked to the AuNPs. In this study, histidine, arginine and cysteine were designed as the functional groups to link citrate-capped AuNPs. Histidine-containing peptides have been studied extensively because of their high affinity to metal. When histidine is added to the citrate-capped AuNPs, the citrate molecules can be partly replaced by histidine through ligand exchange. Then, the imidazole group coordinates the AuNPs through $\mathrm{N} \varepsilon-\mathrm{Au}$ interactions ${ }^{25}$ which not only fix the AuNPs but also prevent the agglomeration of nanoparticles (Scheme 3A). Therefore, CoA$\mathrm{H}$-AuNPs display favorable activity in the reduction of 4-NP. In the case of CoA-R-AuNPs, the positive arginine is directly attached to the negative citrate through electrostatic
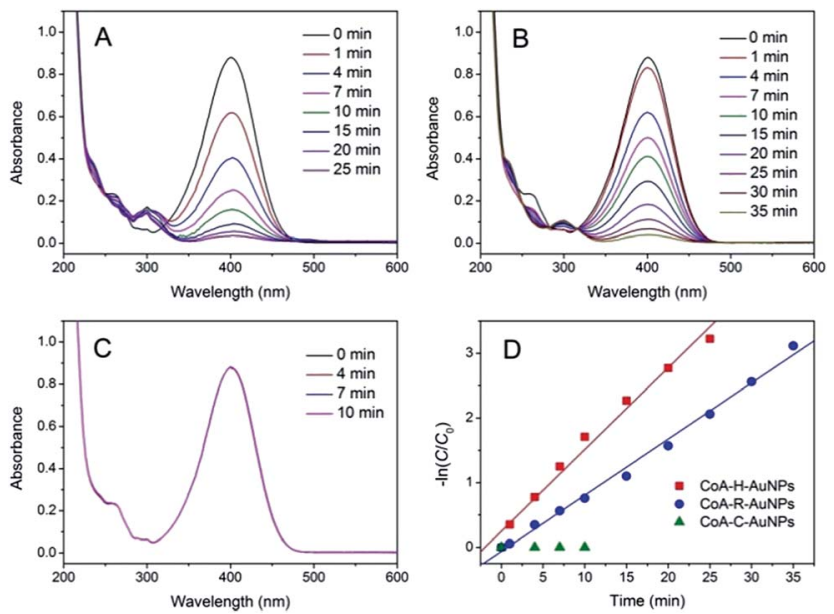

Fig. 4 Time-dependent UV-Vis spectra for the reduction of 4-nitrophenol catalyzed by $\mathrm{CoA}-\mathrm{H}-\mathrm{AuNPs}(\mathrm{A}), \mathrm{CoA}-\mathrm{R}-\mathrm{AuNPs}(\mathrm{B})$, and $\mathrm{CoA}-$ $C-A u N P s(C)$ and their corresponding $-\ln \left(C / C_{0}\right)$ vs. time plots (D). 
A

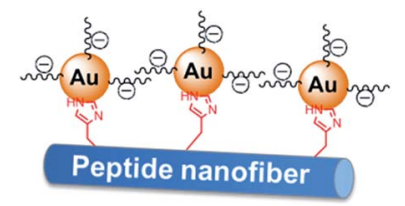

B
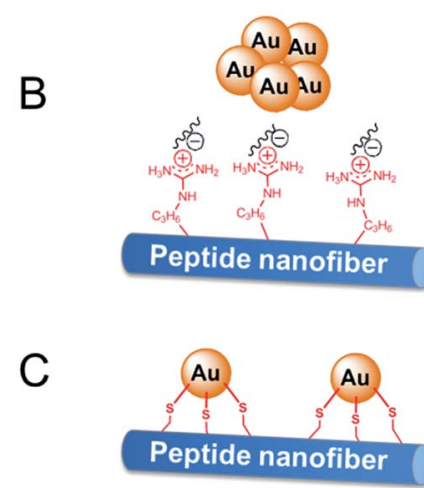

Scheme 3 Immobilization of AuNPs on peptide nanofibers through different amino acid residuals.

interactions, which severely weakens the electrostatic repulsion between AuNPs, leading to their agglomeration. As a result, the agglomerated AuNPs are more liable to leak from the peptide nanofibers, leading to a low AuNPs amount and the lower catalytic activity (Scheme 3B). The covalent S-Au bond between the thiol group on cysteine and the AuNPs is much stronger than $\mathrm{N} \varepsilon-\mathrm{Au}$ and electrostatic interactions. Many reports have demonstrated that the activity of AuNPs is badly damaged because the active sites on the surface of the AuNPs were irreversibly occupied by the formation of multiple $\mathrm{S}-\mathrm{Au}$ bonds (Scheme 3C). As a result, the AuNPs on the cysteine-containing peptide nanofibers lost almost all of their catalytic ability.

Because $\mathrm{NaBH}_{4}$ is present in a significant excess in the reaction, the concentration of $\mathrm{NaBH}_{4}$ is assumed to be constant and the rate of the reaction only depends on the concentration of 4-NP. The reaction rate can be given as:

$$
-r=\frac{-\mathrm{d} C}{\mathrm{~d} t}=k C
$$

where $C$ is the concentration of 4 -NP at time $t$ and $k$ is the reaction rate constant. The rate constant of the pseudo-firstorder reaction can be calculated as:

$$
k=\frac{-\ln \left(\frac{C}{C_{0}}\right)}{t}
$$

Fig. 4D show the kinetic curves for the degradation of 4-NP by the peptide-immobilized AuNPs. A linear relationship between $\ln \left(C / C_{0}\right)$ and the reaction time suggests that the reaction is in agreement with the pseudo-first-order kinetics model, and the rate constants are $k_{\mathrm{CoA}-\mathrm{H}-\mathrm{AuNPs}}=0.127 \mathrm{~min}^{-1}$ and $k_{\mathrm{CoA}-}$ ${ }_{\mathrm{R}-\mathrm{AuNPs}}=0.087 \mathrm{~min}^{-1}$. Compared with free AuNPs $\left(k_{\mathrm{AuNPs}}=\right.$ $0.880 \mathrm{~min}^{-1}$ ) that used for the preparation of peptideimmobilized AuNPs, the activity yield of CoA-H-AuNPs and CoA-R-AuNPs are $14.3 \%$ and $10 \%$, respectively. This result indicated that the activity of CoA-X-AuNPs can be further improved if more AuNPs can be attached to peptide nanofibers and favourably using the histidine-containing peptide.

Congo red is a popular used azo dye in textile, paper and plastic industries which usually causes serious damage for environment. In order to investigate the practical performance of the peptide-immobilized AuNPs, we then determined the degradation of Congo red by using peptide-immobilized AuNPs. As shown in Fig. $\mathrm{S} 2, \uparrow$ the linear relationship between $\ln \left(C / C_{0}\right)$ and the reaction time also indicated that the reaction is in consistent with the pseudo-first-order kinetics model, and the rate constants are $k_{\mathrm{CoA}-\mathrm{H}-\mathrm{AuNPs}}=0.276 \mathrm{~min}^{-1}$ and $k_{\mathrm{CoA}-\mathrm{R}-\mathrm{AuNPs}}$ $=0.089 \mathrm{~min}^{-1}$. Similarly, CoA-C-AuNPs displayed null activity on Congo red. Comparing with AuNPs immobilized on Salmalia malabarica gum $\left(k=0.236 \mathrm{~min}^{-1}\right)^{26}$ or GO-G1PAMAM $(k=0.21$ $\left.\min ^{-1}\right),{ }^{27}$ the as-prepared CoA-H-AuNPs displayed a better catalytic performance in the degradation of Congo red.

\section{The stability and reusability of peptide-immobilized AuNPs}

Stability is an important property for catalysts in industrial applications, especially for nanoscaled catalysts. Agglomeration typically occurs during long reactions, which unfortunately suppresses the activity of the nanocatalyst. Fig. 5 shows the stability of the peptide-immobilized AuNPs under the reaction conditions. CoA-H-AuNPs were found to retain $95 \%$ of its activity after $96 \mathrm{~h}$, implying that the AuNPs are stably immobilized on the peptide nanofibers and maintain a high level of activity during the incubation period. This activity is attributed to the fibrous structure, which provides a robust support upon which to fix the AuNPs, preventing them from agglomerating. However, we found that the activity of CoA-R-AuNPs declined rapidly during the incubation period. CoA-R-AuNPs lost nearly $50 \%$ of its activity in $96 \mathrm{~h}$, which was similar to that observed for the free AuNPs. Fig. S3† shows the images of CoA-H-AuNPs, CoA-R-AuNPs and free AuNPs before and after $72 \mathrm{~h}$ incubation. It can be seen that the CoA-H-AuNPs kept homogeneous hydrogel during the incubation. However, for CoA-R-AuNPs, the reddish brown precipitate became even cloudier which implies the undesired agglomeration of AuNPs during the incubation period. For free AuNPs solution, precipitate can be observed at the bottom of the vessel which implying the low stability of free AuNPs.

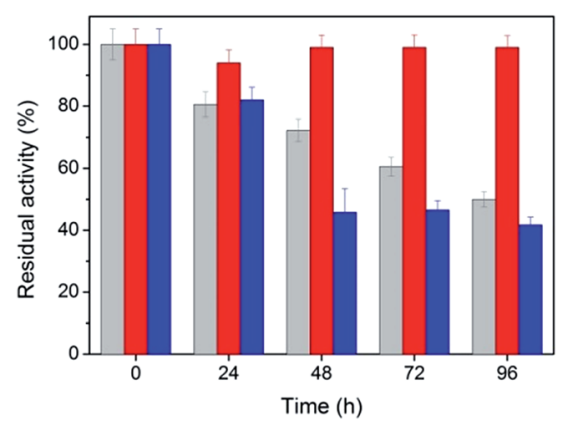

Fig. 5 Stability of $\mathrm{COA}-\mathrm{H}-\mathrm{AuNPs}$ (red), CoA-R-AuNPs (blue) and free AuNPs (grey). 


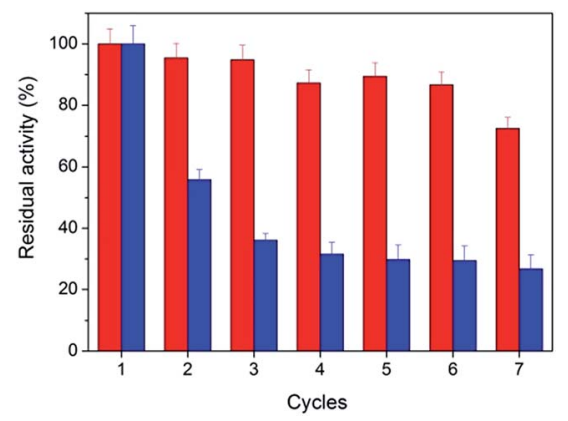

Fig. 6 Reusability of CoA-H-AuNPs (red) and CoA-R-AuNPs (blue).

From the point of saving cost and environmental protection, the reusability is another important parameter for catalysts especially in industrial use. The as-prepared peptideimmobilized AuNPs can be separated from the reaction system through centrifugation or filtration. Fig. 6 shows the reusability of the peptide-immobilized AuNPs under reaction condition. CoA-H-AuNPs could be recycled and reused for at least 7 times with a residual activity of $75 \%$. The decline of activity might due to the gradual mass loss after repeated recycles. However, CoAR-AuNPs lost almost half of its original activity after the first recycle operation, and remained only $29 \%$ after the 7 times. This might due to the low stability of the agglomerated AuNPs in CoA-R-AuNPs compared with that of CoA-H-AuNPs. Therefore, we conclude that histidine-conjuncted peptide nanofibers are a preferable support to immobilize AuNPs.

\section{Conclusions}

In this paper, Fmoc-tripeptides conjuncted with different residues (histidine, arginine and cysteine) were used to immobilize AuNPs through co-assembly. AFM, TEM, UV-Vis and CD analyses demonstrated that the AuNPs can be attached to the peptide nanofibers and do not affect the basic secondary structure of the peptide nanofibers. The activity of peptideimmobilized AuNPs mostly depends on the type of residue that the AuNPs are bound to. CoA-H-AuNPs exhibited favorable activity and stability in the reduction of 4-NP and azo dye as a result of the formation of stable $\mathrm{N} \varepsilon-\mathrm{Au}$ interactions, which not only fix the AuNPs but also prevent agglomeration of the nanoparticles.

\section{Acknowledgements}

This work was supported by the Ministry of Science and Technology of China (2012YQ090194, 2013AA102204), the Natural Science Foundation of China (21676191, 21476165, 21621004) and the Natural Science Foundation of Tianjin (13JCQNJC09300).

\section{References}

1 V. Polshettiwar and R. S. Varma, Green Chem., 2010, 12, 743754.
2 W. Xu, J. S. Kong, Y.-T. E. Yeh and P. Chen, Nat. Mater., 2008, 7, 992-996.

3 R. Schlögl and S. B. Abd Hamid, Angew. Chem., Int. Ed., 2004, 43, 1628-1637.

4 M. Wang, W. Qi, R. Su and Z. He, Chem. Eng. Sci., 2015, 135, 21-32.

5 M.-C. Daniel and D. Astruc, Chem. Rev., 2004, 104, 293-346. 6 Y. Liu, H. Dai, J. Deng, S. Xie, H. Yang, W. Tan, W. Han, Y. Jiang and G. Guo, J. Catal., 2014, 309, 408-418.

7 Q. He, S. J. Freakley, J. K. Edwards, A. F. Carley, A. Y. Borisevich, Y. Mineo, M. Haruta, G. J. Hutchings and C. J. Kiely, Nat. Commun., 2016, 7, 12905.

8 D. Li, J.-F. Wehrung and Y. Zhao, J. Mater. Chem. A, 2015, 3, 5176-5182.

9 Y. Qu, W. Shen, X. Pei, F. Ma, S. You, S. Li, J. Wang and J. Zhou, J. Environ. Sci., 2016, DOI: 10.1016/j.jes.2016.09.007.

10 H. Wang, Z. Dong and C. Na, ACS Sustainable Chem. Eng., 2013, 1, 746-752.

11 D. M. Dotzauer, J. Dai, L. Sun and M. L. Bruening, Nano Lett., 2006, 6, 2268-2272.

12 X. Fang, H. Ma, S. Xiao, M. Shen, R. Guo, X. Cao and X. Shi, J. Mater. Chem., 2011, 21, 4493-4501.

13 T. Niu, J. Xu, W. Xiao and J. Huang, RSC Adv., 2014, 4, 49014904.

14 L. Lin, W. Wu, J. Huang, D. Sun, N. u. M. Waithera, Y. Zhou, H. Wang and Q. Li, Chem. Eng. J., 2013, 225, 857-864.

15 S. Fleming and R. V. Ulijn, Chem. Soc. Rev., 2014, 43, 81508177.

16 Q. Li, M. Chen, D. Chen and L. Wu, Chem. Mater., 2016, 28, 6584-6590.

17 X. Yan, Y. Cui, Q. He, K. Wang and J. Li, Chem. Mater., 2008, 20, 1522-1526.

18 Y. Wang, Z. Zhang, L. Xu, X. Li and H. Chen, Colloids Surf., B, 2013, 104, 163-168.

19 A. N. Rissanou, E. Georgilis, E. Kasotakis, A. Mitraki and V. Harmandaris, J. Phys. Chem. B, 2013, 117, 3962-3975.

20 R. Orbach, L. Adler-Abramovich, S. Zigerson, I. MironiHarpaz, D. Seliktar and E. Gazit, Biomacromolecules, 2009, 10, 2646-2651.

21 J. M. Kogot, H. J. England, G. F. Strouse and T. M. Logan, J. Am. Chem. Soc., 2008, 130, 16156-16157.

22 Y. Zhang, J. Jiang, M. Li, P. Gao, Y. Zhou, G. Zhang, S. Shuang and C. Dong, Talanta, 2016, 161, 520-527.

23 V. Jayawarna, M. Ali, T. A. Jowitt, A. F. Miller, A. Saiani, J. E. Gough and R. V. Ulijn, Adv. Mater., 2006, 18, 611-614.

24 B. Ding, Y. Li, M. Qin, Y. Ding, Y. Cao and W. Wang, Soft Matter, 2013, 9, 4672-4680.

25 J. K. Lim, Y. Kim, S. Y. Lee and S.-W. Joo, Spectrochim. Acta, Part A, 2008, 69, 286-289.

26 B. R. Ganapuram, M. Alle, R. Dadigala, A. Dasari, V. Maragoni and V. Guttena, Int. Nano Lett., 2015, 5, 215-222.

27 R. Rajesh, S. S. Kumar and R. Venkatesan, New J. Chem., 2014, 38, 1551-1558. 